\title{
Main Components of Liquid Biopsy are Greater than it Seems?
}

\author{
Fatemeh Khatami and Seyed Mohammad Tavangar* \\ Tehran University of Medical Science, Iran
}

Received: December 22, 2017; Published: January 18, 2018

*Corresponding author: Seyed Mohammad Tavangar, Department of Pathology, Dr. Shariati Hospital, Tehran University of Medical Sciences, Tehran, Iran

\begin{abstract}
Abbreviations: CTCs: Circulating Tumor Cells; NGS: Next Generation Sequencing; ecDNA: Circular Extra Chromosomal DNA; cfDNA: Cell Frees
\end{abstract} DNA

\section{Editorial}

Long time ago it was wrongly thought that tumor genes and cells are only existence in the exact tumor site. In spite of the fact of the hypothesis that circulating tumor cells (CTCs) are a fundamental prerequisite to metastasis ( first projected in the 1896 by Thomas Ashworth, an Australian pathologist, ) and the presence of cfDNA report in human plasma by Mandel and Metals in 1948, liquid biopsy were totally ignored till 1977. In 1977, researchers made the novel observation that cancer patients carried cell-free DNA in their peripheral blood which was Initial progress on further of liquid biopsy. Without a doubt, significant progress was not made until recent years with the advent of Next Generation Sequencing (NGS) technology, which significantly improved the sensitivity and specificity of ctDNA detection. Interestingly, research in this field of liquid biopsy has entered a "golden age" in which the huge potential of liquid biopsy main components including CTCs, cfDNA and exosomes make tumor diagnosis and treatment much clearer than before. Liquid biopsy tests are fast traction as a viable substitute to traditional diagnostic tests for cancer. It has the potential to facilitate detect cancer at earlier stages, present a less-expensive and less-invasive way to monitor patients throughout treatment, and can help doctors make better decisions about which drugs are the best fit for personal patients.

The questions are now:

a) We know all about liquid biopsy components?

b) Is there any possibility that we still are missing a main representative of tumor genetics? c) If the answer is yes what could be that components: RNA, DNA, or cell?

Answering to these questions is not easy but it is supposed to keep this question in mind. By way of illustration, in molecular biology covalently closed circular DNAs are able to pass through double layer of eukaryotic cellular membrane. Very recently the presence of circular extra chromosomal DNA (ecDNA) has been shown by Turner KM [1] which is different in seventeen different types of cancers. In fact, ecDNA are the tricky way of oncogenes to increase their copy number. It can be imagine that the presence of ecDNA in the blood of cancer patients as a subpopulation of liquid biopsy can be possible. On the occasion of their presence in blood they will be very beneficial to cover the small amount of cell frees DNA (cfDNA). Isolation and characterization of ecDNA will be possible by a sensitive method entitled Circle-Seq. The origin of tumor more than its prognosis and diagnosis will be possible in the easiest way by using ecDNA as a liquid biopsy component. Thanks to the latest breakthroughs in gene sequencing techniques all liquid biopsy components will be discovered and determined within next decade.

\section{References}

1. Kristen M. Turner, Viraj Deshpande, Doruk Beyter, Tomoyuki Koga, Jessica Rusert, et al. (2017) Extrachromosomal oncogene amplification drives tumour evolution and genetic heterogeneity. Nature 543: 122125. 
CC (i) This work is licensed under Creative Commons Attribution 4.0 License

DOI: 10.32474/OAJOM.2018.01.000102

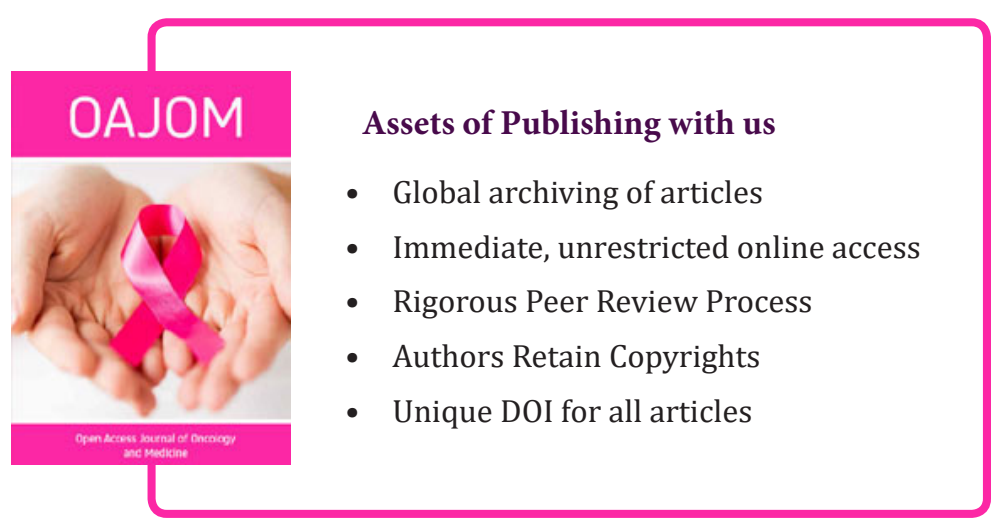

